\title{
COMPARISON OF NUMERICAL AND ANALYTICAL APPROXIMATIONS OF THE EARLY EXERCISE BOUNDARY OF AMERICAN PUT OPTIONS
}

\author{
M. LAUKO ${ }^{1}$ and D. ŠEVČOVIČ ${ }^{\bowtie 1}$ \\ (Received 7 February, 2010; revised 9 September, 2010)
}

\begin{abstract}
We present qualitative and quantitative comparisons of various analytical and numerical approximation methods for calculating a position of the early exercise boundary of American put options paying zero dividends. We analyse the asymptotic behaviour of these methods close to expiration, and introduce a new numerical scheme for computing the early exercise boundary. Our local iterative numerical scheme is based on a solution to a nonlinear integral equation. We compare numerical results obtained by the new method to those of the projected successive over-relaxation method and the analytical approximation formula recently derived by Zhu ['A new analytical approximation formula for the optimal exercise boundary of American put options', Int. J. Theor. Appl. Finance 9 (2006) 1141-1177].
\end{abstract}

2000 Mathematics subject classification: primary 35R35; secondary 91B28, $62 \mathrm{P} 05$.

Keywords and phrases: option pricing, American put option, early exercise boundary, limiting behaviour close to expiry.

\section{Introduction}

The analysis of the early exercise boundary and the optimal stopping time for American put options on assets paying zero dividends has attracted a lot of attention from both theoretical as well as practical points of view. An American put option is a financial contract between the writer and the holder of the option. It gives the holder the right, but not the obligation, to sell the underlying asset at the prescribed strike price at any time before expiration. Under the standard assumptions made on the underlying stock process and completeness of the financial market [17, 21], American put options can be priced using the Black-Scholes equation [4] on a time-dependent domain of the underlying asset price. More precisely, the early exercise boundary problem for

\footnotetext{
${ }^{1}$ Department of Applied Mathematics \& Statistics, Comenius University, 84248 Bratislava, Slovakia; e-mail: lauko5@st.fmph.uniba.sk, sevcovic@fmph.uniba.sk.

(C) Australian Mathematical Society 2010, Serial-fee code 1446-1811/2010 \$16.00
} 
American put options can be formulated as follows: find a solution $V=V(S, t)$ and the early exercise boundary position $S_{f}=S_{f}(t)$ satisfying

$$
\begin{gathered}
\frac{\partial V}{\partial t}+r S \frac{\partial V}{\partial S}+\frac{\sigma^{2}}{2} S^{2} \frac{\partial^{2} V}{\partial S^{2}}-r V=0, \quad 0<t<T, S_{f}(t)<S<\infty \\
V(+\infty, t)=0, \quad V\left(S_{f}(t), t\right)=E-S_{f}(t), \quad \frac{\partial V}{\partial S}\left(S_{f}(t), t\right)=-1 \\
V(S, T)=(E-S)^{+}
\end{gathered}
$$

The solution $V(S, t)$ is defined on a time-dependent domain $S \in\left(S_{f}(t), \infty\right)$, where $t \in(0, T)$ (see Kwok [21]). Here $S>0$ stands for the underlying stock price, $E>0$ is the exercise (strike) price, $r>0$ is the risk-free rate, $\sigma>0$ is the volatility of the underlying stock process and $T$ denotes the time of maturity. In what follows, we denote by $\tau=T-t$ the time to maturity. The function $[0, T] \ni t \mapsto S_{f}(t) \in \mathbb{R}$ represents the early exercise boundary position. The free boundary problem (1.1) is a basis for development of various integral equations for describing the early exercise boundary position $S_{f}(t)$. The analytical approximation formulae for $S_{f}$ are often based on approximation of a solution to such an integral equation. Notice that there are also other numerical methods for approaching the free boundary problem (1.1), for example front-fixing and transformation methods. We refer the reader to Kwok and Wu [22], Ševčovič [26, 27], Ankudinova and Ehrhardt [2], Ehrhardt and Mickens [10] and references therein. Following Kwok [21], a solution $V=V(S, t)$ to the problem of pricing American put options fulfils the following variational inequality:

$$
\begin{aligned}
& \frac{\partial V}{\partial t}+r S \frac{\partial V}{\partial S}+\frac{\sigma^{2}}{2} S^{2} \frac{\partial^{2} V}{\partial S^{2}}-r V \leq 0, \quad V(S, t) \geq V(S, T) \\
& \left(\frac{\partial V}{\partial t}+r S \frac{\partial V}{\partial S}+\frac{\sigma^{2}}{2} S^{2} \frac{\partial^{2} V}{\partial S^{2}}-r V\right)(V(S, t)-V(S, T))=0 \\
& \quad \text { for all } 0<t<T, 0<S<\infty \\
& V(0, t)=E, \quad V(+\infty, t)=0 \text { for } 0<t<T \\
& V(S, T)=(E-S)^{+} \text {for } 0<S<\infty
\end{aligned}
$$

The formulation (1.2) of the problem of pricing the American put option is often used when we need to compute not only the free boundary position $S_{f}(t)$ but also the solution $V(S, t)$. The above variational inequality can be effectively solved by means of the so-called projected successive over-relaxation (PSOR) method of Elliot and Ockendon [13].

In the last two decades, many different, but equivalent, integral equations for pricing American put options have been derived by Barone-Adesi and Whaley [3], Bunch and Johnson [5], Carr et al. [6], MacMillan [23] and others. Asymptotic analysis often leads to an approximate expression for the free boundary close to expiry. Since a closed-form analytical formula for the early exercise boundary position is not known, many authors have investigated various approximation models and derived different approximate 
expressions for valuing American call and put options (see, for example, Geske and Johnson [15], Geske and Roll [16], Johnson [18], Karatzas [19], Kuske and Keller [20], Evans et al. [14], Mynemi [25] and recent papers by Alobaidi et al. [1], Mallier and Alobaidi [24], Stamicar et al. [28], the survey paper by Chadam [7] and references therein). We also refer to the books by Kwok [21] and Wilmott et al. [29] for a survey of classical theoretical and computational results on pricing of American put options.

In this paper, we focus on comparison of the valuation formulae due to Evans, Kuske and Keller [14, 20], Stamicar et al. [28] and the recent analytical approximation formula by Zhu [30] (see also [31, 32]). Our main goal is to present qualitative and quantitative comparisons of the aforementioned analytical and numerical approximation methods for calculating the early exercise boundary position. First, we analyse and compare the asymptotic behaviour of the early exercise boundary close to expiry for analytical approximations developed by the aforementioned authors. We show that the approximations due to Evans, Kuske and Keller and Stamicar et al. have the same asymptotic behaviour of $S_{f}(t)$ as $t \rightarrow T$, and that the approximation due to Zhu has asymptotic behaviour differing from these by a logarithmic factor. Although Zhu [30] pointed out that his formula did not behave correctly near expiry, a quantitative analysis of the asymptotic behaviour was not given. We present a quantitative order analysis of Zhu's approximation near the expiry in the first part of this paper.

In the second part of the paper, we propose a new local iterative numerical scheme for computation of $S_{f}(t), t \in[0, T]$, based on a solution to the nonlinear integral equation from [28]. We compare numerical results obtained by the new method to those of the projected successive over-relaxation method of Elliot and Ockendon [13] for solving the variational inequality (1.2), and the approximation recently developed by Zhu [30] (see also [31, 32]).

\section{Analytical approximate valuation formulae}

In this section we present a survey of analytical, implicit integral and numerical approximation schemes for computing the early exercise boundary for American put options. First we focus on the recent result due to Zhu [30] who derived a closed-form analytical approximation formula for the early exercise boundary position $S_{f}(t)=\varrho(T-t)$. We also derive the asymptotic behaviour of $S_{f}(t)$ for $t \rightarrow T$. Next we concentrate on implicit representation formulae for $\varrho(\tau)$ expressed in the form of a single nonlinear integral equation for the function $\varrho$. We recall the implicit integral equation derived by Stamicar et al. [28]. We again derive the asymptotic behaviour of the early exercise boundary position as $t \rightarrow T$. In the last subsection we present another approximation derived by Evans, Kuske and Keller [14, 20].

2.1. Approximation due to $\mathrm{Zhu} Z \mathrm{Zhu}$ [30] applied Laplace transforms to a dimensionless partial differential equation and obtained a closed-form analytical approximation formula for the early exercise boundary position as a sum of a perpetual option and an integral that evaluates the early exercise boundary position. 
The resulting formula for the early exercise boundary $S_{f}(t)=\varrho(T-t)$ reads as follows:

$$
\varrho^{\mathrm{Zhu}}(\tau)=\frac{\gamma E}{1+\gamma}+\frac{2 E}{\pi} \int_{0}^{\infty} \frac{\zeta e^{-\tau \sigma^{2}\left(a^{2}+\zeta^{2}\right) / 2}}{a^{2}+\zeta^{2}} e^{-f_{1}^{*}(\zeta)} \sin \left(f_{2}^{*}(\zeta)\right) d \zeta
$$

where $\gamma=2 r / \sigma^{2}, a=(1+\gamma) / 2, b=(1-\gamma) / 2$, and

$$
\begin{aligned}
& f_{1}^{*}(\zeta)=\frac{1}{b^{2}+\zeta^{2}}\left[b \ln \left(\frac{1}{\gamma} \sqrt{a^{2}+\zeta^{2}}\right)+\zeta \arctan (\zeta / a)\right] \\
& f_{2}^{*}(\zeta)=\frac{1}{b^{2}+\zeta^{2}}\left[\zeta \ln \left(\frac{1}{\gamma} \sqrt{a^{2}+\zeta^{2}}\right)-b \arctan (\zeta / a)\right] .
\end{aligned}
$$

Notice that the first summand in (2.1) represents the constant value of a perpetual put option, that is, the $\operatorname{limit}_{\tau \rightarrow \infty} \varrho(\tau)=\gamma E /(1+\gamma)$.

Early exercise boundary asymptotics close to expiry Next we examine the asymptotic behaviour of the function $\varrho^{\text {Zhu }}(\tau)$ for $\tau \rightarrow 0$. Notice that we have $\varrho(0)=$ $S_{f}(T)=E$ (see Kwok [21]). We shall prove that

$$
\lim _{\tau \rightarrow 0^{+}} \frac{E-\varrho^{\mathrm{Zhu}}(\tau)}{\sqrt{\tau}(-\ln \tau)}=\frac{1}{\sqrt{2 \pi}} E \sigma .
$$

Indeed, if we introduce the change of variable $s=\tau \sigma^{2}\left(a^{2}+\zeta^{2}\right) / 2$ we obtain

$$
\frac{E-\varrho^{\mathrm{Zhu}}(\tau)}{\sqrt{\tau}(-\ln \tau)}=\frac{2 E}{\pi} \int_{\tau \sigma^{2} a^{2} / 2}^{\infty} \frac{1-e^{-s}}{2 s} e^{-f_{1}^{*}} \frac{\sin \left(f_{2}^{*}\right)}{\sqrt{\tau}(-\ln \tau)} d s \quad \text { for any } \tau \in(0, T],
$$

where $f_{i}^{*}=f_{i}^{*}\left(\left(2 s /\left(\tau \sigma^{2}\right)-a^{2}\right)^{1 / 2}\right), i=1,2$. It is easy to verify that

$$
\begin{aligned}
\lim _{\tau \rightarrow 0^{+}} f_{1}^{*} & =0, \quad \lim _{\tau \rightarrow 0} f_{2}^{*}=0, \\
\lim _{\tau \rightarrow 0^{+}} \frac{\sin \left(f_{2}^{*}\right)}{\sqrt{\tau}(-\ln \tau)} & =\lim _{\tau \rightarrow 0} \frac{f_{2}^{*}}{\sqrt{\tau}(-\ln \tau)}=\frac{\sigma}{2 \sqrt{2 s}},
\end{aligned}
$$

for any $s>0$. Using the Lebesgue dominated convergence theorem, we finally obtain

$$
\lim _{\tau \rightarrow 0^{+}} \frac{E-\varrho^{\mathrm{Zhu}}(\tau)}{\sqrt{\tau}(-\ln \tau)}=\frac{E \sigma}{\pi} \int_{0}^{\infty} \frac{1-e^{-s}}{(2 s)^{3 / 2}} d s=\frac{1}{\sqrt{2 \pi}} E \sigma
$$

as claimed. As a consequence we obtain the following asymptotic approximation to the formula of Zhu:

$$
\varrho^{\mathrm{Zhu}}(\tau) \approx E\left(1-\frac{\sigma}{\sqrt{2 \pi}} \sqrt{\tau}(-\ln \tau)\right) \text { for } 0<\tau \ll 1,
$$

that is,

$$
\varrho^{\mathrm{Zhu}}(\tau)=E\left(1-\frac{\sigma}{\sqrt{2 \pi}} \sqrt{\tau}(-\ln \tau)\right)+o(\sqrt{\tau}(-\ln \tau)) \quad \text { as } \tau \rightarrow 0^{+} .
$$

In Figure 1 we present a comparison of the analytical solution $\varrho^{\text {Zhu }}(\tau)$ and its asymptotic approximation (2.3) for $\tau \in[0, T]$ and $E=100, \sigma=0.3, r=0.1, T=10^{-4}$. 


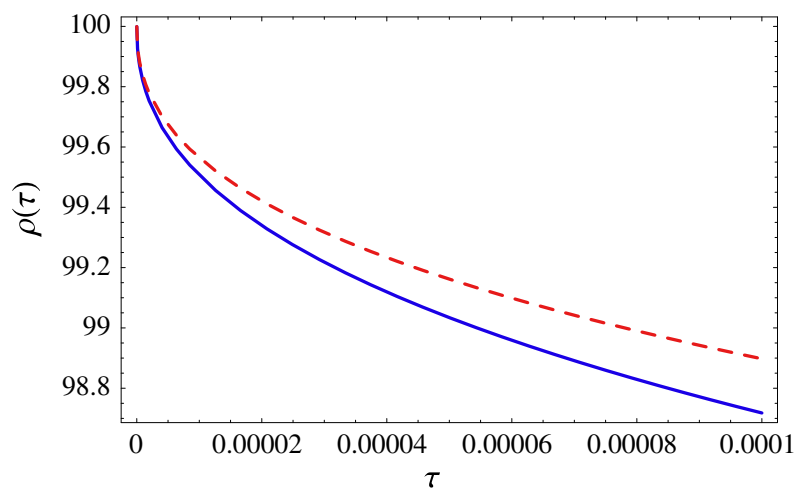

FIgURE 1. Comparison of the analytical solution $\varrho^{\text {Zhu }}$ (solid curve) and its asymptotic approximation (2.3) (dashed curve).

Convexity of the early exercise boundary One of the important features of the early exercise boundary for American put options is the convexity of the function $\varrho(\tau)=S_{f}(T-\tau)$ for $\tau \in(0, T]$. An analytical proof of the convexity of $\varrho$ was recently presented by Chen et al. [9]. We also recall that the early exercise boundary is log-concave as a function of the logarithm of the underlying asset price (see Ekström [11] and Ekström and Tysk [12]).

A relatively simple proof of the convexity of $\varrho=\varrho^{\text {Zhu }}$ follows directly from the analytical valuation formula (2.1). Indeed, for any $0<\tau \leq T$, we have the following expression for the second derivative of the function $\varrho^{\mathrm{Zhu}}(\tau)$ :

$$
\frac{d^{2}}{d \tau^{2}} \varrho^{\mathrm{Zhu}}(\tau)=\frac{2 E \sigma^{4}}{4 \pi} \int_{0}^{\infty}\left(a^{2}+\zeta^{2}\right) \zeta e^{-\tau \sigma^{2}\left(a^{2}+\zeta^{2}\right) / 2} e^{-f_{1}^{*}(\zeta)} \sin \left(f_{2}^{*}(\zeta)\right) d \zeta
$$

In what follows, we shall prove that $f_{2}^{*}(\zeta) \equiv f_{2}^{*}(\zeta ; \gamma) \in[0, \pi]$ provided that $\gamma \geq \gamma_{0}$, where $\gamma_{0}>0$ is a constant given by

$$
\gamma_{0}=\min \left\{\gamma>0 \mid \max _{\zeta>0} f_{2}^{*}(\zeta, \gamma) \leq \pi\right\}
$$

The numerical value of $\gamma_{0}$ can be estimated as $\gamma_{0} \approx 0.0167821$.

Corollary 2.1. If $2 r / \sigma^{2}=\gamma \geq \gamma_{0}$, where $\gamma_{0} \approx 0.0167821$, then $f_{2}^{*}=f_{2}^{*}(\zeta, \gamma) \in$ $[0, \pi]$ for any $\zeta>0$. As a consequence, the second derivative of $\varrho^{\mathrm{Zhu}}$ is positive, that $i$, the function $Q^{\mathrm{Zhu}}(\tau)$ as well as the early exercise boundary $S_{f}(t)$ for the American put option are convex functions.

REMARK 2.2. Notice that the condition $2 r / \sigma^{2}=\gamma \geq \gamma_{0}$ is fulfilled for typical market-based choices of the model parameters $r$ and $\sigma$. For example, if $r=0.01$ (that is, $r=1 \%$ per annum) then $2 r / \sigma^{2} \geq \gamma_{0}$ provided that the condition $\sigma^{2}<1.19$ (that is, $\sigma^{2} \leq 119 \%$ p.a.) is satisfied. In Figure 2 we present graphs of the function 

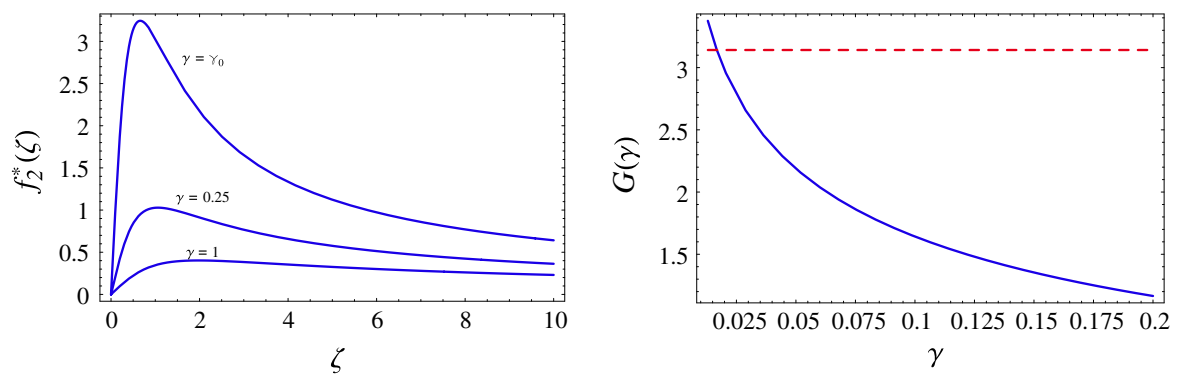

FIGURE 2. The function $f_{2}^{*}=f_{2}^{*}(\zeta ; \gamma)$ for various values of the parameter $\gamma$ (left) and the function $G(\gamma)=\max _{\zeta>0} f_{2}^{*}(\zeta ; \gamma)$ (right).

$\zeta \mapsto f_{2}^{*}(\zeta ; \gamma)$ for various values of the parameter $\gamma$, including the critical value $\gamma=\gamma_{0} \approx 0.0167821$ for which the function $G(\gamma)=\max _{\zeta>0} f_{2}^{*}(\zeta ; \gamma)$ attains the critical value $G\left(\gamma_{0}\right)=\pi$.

2.2. Approximation due to Stamicar et al. In [28] Stamicar et al. derived a single nonlinear integral equation for the early exercise boundary position. Based on this equation they derived an improved analytical approximation for the free boundary near the expiry. The asymptotic behaviour and justification of the early exercise behaviour close to expiry have recently been analysed by Chen et al. [9] and Chen and Chadam [8]. They proved that the correct asymptotic expansion can be obtained from the nonlinear integral equation developed by Stamicar et al. [28]. We briefly recall the key steps of the derivation of the nonlinear integral equation for the early exercise boundary position $\varrho(\tau)=S_{f}(T-\tau)$ for the free boundary problem (1.1). Let us introduce the change of variables $x=\ln (S / \varrho(\tau))$ where $\tau=T-t, \varrho(\tau)=$ $S_{f}(T-\tau)$. Similarly to the case of a call option (see [26]), we define a synthesized portfolio $\Pi$ for the put option by

$$
\Pi(x, \tau)=V(S, t)-S \frac{\partial V}{\partial S}(S, t) .
$$

It is easy to verify that $\Pi$ is a solution to the following parabolic equation:

$$
\begin{gathered}
\frac{\partial \Pi}{\partial \tau}-a(\tau) \frac{\partial \Pi}{\partial x}-\frac{\sigma^{2}}{2} \frac{\partial^{2} \Pi}{\partial x^{2}}+r \Pi=0, \quad x>0, \tau \in(0, T), \\
\Pi(0, \tau)=E, \quad \Pi(\infty, \tau)=0, \quad \Pi(x, 0)=0, \quad x>0, \tau \in(0, T), \\
\frac{\sigma^{2}}{2} \frac{\partial \Pi}{\partial x}(0, \tau)=-r E \quad \text { for } \tau \in(0, T),
\end{gathered}
$$

where $a(\tau)=\dot{\varrho}(\tau) / \varrho(\tau)+r-\sigma^{2} / 2$ (see Stamicar et al. [28], or Ševčovič [26, 27]). Applying the Fourier transform, one can find the Fourier image of $\Pi$ in terms of the free boundary position $\varrho$. The resulting equation for $\varrho$ reads

$$
\frac{\sigma^{2}}{2} \frac{\partial \Pi}{\partial x}(0, \tau)=-r E,
$$


from which the weakly singular integral equation for $\varrho$ can be found by using the inverse Fourier transform (see [28] for details). More precisely, the function $\varrho(\tau)$ satisfies the equation

$$
\varrho(\tau)=E e^{-\left(r-\sigma^{2} / 2\right) \tau+\sigma \sqrt{2 \tau} \eta(\tau)},
$$

where the auxiliary function $\eta(\tau)$ is a solution to the nonlinear integral equation

$$
\eta(\tau)=-\sqrt{-\ln \left[\frac{r \sqrt{2 \pi \tau}}{\sigma} e^{r \tau}\left(1-\frac{F_{\eta}(\tau)}{\sqrt{\pi}}\right)\right]} \text { for } \tau \in[0, T] .
$$

Here the function $F_{\eta}$ depends on $\eta$ via the expression

$$
\begin{gathered}
F_{\eta}(\tau)=2 \int_{0}^{\pi / 2} e^{-r \tau \cos ^{2} \theta-g_{\eta}^{2}(\tau, \theta)}\left(\frac{\sigma \sqrt{\tau}}{\sqrt{2}} \sin \theta+g_{\eta}(\tau, \theta) \tan \theta\right) d \theta, \\
g_{\eta}(\tau, \theta)=\frac{1}{\cos \theta}\left[\eta(\tau)-\eta\left(\tau \sin ^{2} \theta\right) \sin \theta\right],
\end{gathered}
$$

for $\tau \in[0, T], \theta \in[0, \pi / 2]$. According to [28], the asymptotic analysis of the above integral equation for the unknown function $\eta(\tau)$ enables us to deduce the asymptotic approximation formula for $\eta(\tau)$ as $\tau \rightarrow 0$. The early exercise behaviour of $\varrho(\tau)$ for $\tau \rightarrow 0$ can be then deduced from second-order iteration of the system (2.6) and (2.7) starting with the initial guess $\eta_{0}(\tau)=\left(r-\sigma^{2} / 2\right) \sqrt{\tau} /(\sigma \sqrt{2})$ corresponding to the constant early exercise boundary $S_{f 0}(t) \equiv E$. One can iteratively compute $F_{\eta_{0}}, \eta_{1}$ and $F_{\eta_{1}}, \eta_{2}$. It turns out (see [28]) that the second consecutive iterate $\eta_{2}$ is the lowest order (in $\tau$ ) approximation of $\eta$. Namely,

$$
\eta(\tau) \sim-\sqrt{-\ln \left[\frac{2 r}{\sigma} \sqrt{2 \pi \tau} e^{r \tau}\right]} \text { as } \tau \rightarrow 0^{+} .
$$

Interestingly enough, it has recently been shown by Chen et al. [9] that the early exercise boundary function $\varrho$ is convex (see also [7, 8]). Moreover, the approximation formula (2.9) derived by Stamicar et al. [28] provides the correct asymptotic behaviour for $\tau \rightarrow 0^{+}$. Furthermore, Chen and Chadam [8] derived the sixth-order expansion of the function,

$$
\alpha(\tau)=-\xi-\frac{1}{2 \xi}+\frac{1}{8 \xi^{2}}+\frac{17}{24 \xi^{3}}-\frac{51}{64 \xi^{4}}-\frac{287}{120 \xi^{5}}+\frac{199}{32 \xi^{6}}+O\left(\xi^{-7}\right),
$$

for $\xi=\ln \sqrt{8 \pi r^{2} \tau / \sigma^{2}} \rightarrow-\infty$ as $\tau \rightarrow 0^{+}$where

$$
\varrho(\tau)=E e^{-\sigma \sqrt{2 \tau \alpha(\tau)}} .
$$

Early exercise boundary asymptotics close to expiry As in the case of the analytical approximation formula of Zhu, we examine the asymptotic behaviour of 
the function $\varrho(\tau)$ for $\tau \rightarrow 0$, where $\varrho(\tau) \equiv \varrho^{\mathrm{SSCh}-\mathrm{A}}(\tau)$ is given by the equation

$$
\varrho(\tau)=E e^{-\left(r-\sigma^{2} / 2\right) \tau+\sigma \sqrt{2 \tau} \tilde{\eta}(\tau)} \quad \text { with } \tilde{\eta}(\tau)=-\sqrt{-\ln \left[\frac{2 r}{\sigma} \sqrt{2 \pi \tau} e^{r \tau}\right]} .
$$

Employing expression (2.12), it is straightforward to verify that

$$
\lim _{\tau \rightarrow 0^{+}} \frac{E-\varrho^{\mathrm{SSCh}-\mathrm{A}}(\tau)}{\sqrt{\tau} \sqrt{-\ln \tau}}=E \sigma .
$$

Again, as a consequence of the above limit, we deduce the following asymptotic approximation to the analytical valuation formula due to Stamicar et al.:

$$
\varrho^{\mathrm{SSCh}-\mathrm{A}}(\tau) \approx E(1-\sigma \sqrt{\tau} \sqrt{-\ln \tau}) \text { for } 0<\tau \ll 1,
$$

that is,

$$
\varrho^{\mathrm{SSCh}-\mathrm{A}}(\tau)=E(1-\sigma \sqrt{\tau} \sqrt{-\ln \tau})+o(\sqrt{\tau} \sqrt{-\ln \tau}) \quad \text { as } \tau \rightarrow 0^{+} .
$$

Notice that the asymptotic formula (2.13) differs from the one obtained from Zhu's formula (2.3) by a logarithmic factor $\sqrt{-\ln \tau}$. This could be a direct consequence of the "pseudo-steady-state" approximation made in obtaining the two boundary conditions in [30, Equation (2.11)]. The order analysis presented here quantifies the order of errors that could potentially arise from Zhu's approximation near expiry.

2.3. Approximation due to Evans, Kuske and Keller Kuske and Keller [20] proposed another analytical approximation to the early exercise boundary for times close to expiration. Then, in their paper with Evans [14], they improved and extended the formula for the case of a dividend-paying asset.

We begin with the approximation formula of Kuske and Keller [20] for the position of the early exercise boundary close to expiry $t \rightarrow T$, which reads as follows:

$$
\varrho^{\mathrm{KK}}(\tau) \approx E\left(1-\sigma \sqrt{2 \tau} \sqrt{-\ln \left[\frac{2 r}{\sigma} \sqrt{\frac{9 \pi \tau}{2}}\right]}\right) \text { as } \tau \rightarrow 0^{+} .
$$

Evans et al. [14] derived an improved asymptotic formula:

$$
\varrho^{\mathrm{EKK}}(\tau) \approx E\left(1-\sigma \sqrt{2 \tau} \sqrt{-\ln \left[\frac{2 r}{\sigma} \sqrt{2 \pi \tau}\right]}\right) \text { as } \tau \rightarrow 0^{+} .
$$

Although (2.14), (2.15) and the formula (2.12) of Stamicar et al. differ in higher-order terms of $\tau$,

$$
\lim _{\tau \rightarrow 0^{+}} \frac{E-\varrho^{\operatorname{SSCh}-\mathrm{A}}(\tau)}{\sqrt{\tau} \sqrt{-\ln \tau}}=\lim _{\tau \rightarrow 0^{+}} \frac{E-\varrho^{\mathrm{KK}}(\tau)}{\sqrt{\tau} \sqrt{-\ln \tau}}=\lim _{\tau \rightarrow 0^{+}} \frac{E-\varrho^{\mathrm{EKK}}(\tau)}{\sqrt{\tau} \sqrt{-\ln \tau}}=E \sigma .
$$

This means that the approximation formulae due to Evans, Kuske and Keller [14, 20] and Stamicar et al. [28] have the same asymptotic behaviour close to expiry $t \approx T$, that is, for $0<\tau \ll 1$. 


\section{Numerical methods}

The early exercise boundary function $\varrho(\tau)$ for the time interval $\tau \in[0, T]$ can also be approximated by using numerical methods. In this section we present two approaches: a new local iterative algorithm based on the integral equation due to Stamicar et al. [28], and the well-known PSOR method (see Kwok [21]).

3.1. A new numerical algorithm We introduce a new numerical algorithm for computation of the early exercise boundary of American put options. This is based on a solution to the system of implicit equations (2.6)-(2.8) derived by Stamicar et al. [28]. The idea of the proposed algorithm is to sequentially compute values of the auxiliary function $\eta=\eta(\tau)$ at nodal points $\tau_{i} \in[0, T]$. In our new local iterative algorithm we only have to find a root of a real-valued function at each nodal point $\tau_{i}$. This is due to the form of the functions $F_{\eta}, g_{\eta}$ (see (2.7) and (2.8)) whose values at $\tau \in(0, T]$ depend only on the value $\eta(\tau)$ and the history path $\{\eta(\xi), 0 \leq \xi<\tau\}$. Our new algorithm for approximating the early exercise boundary $\varrho(\tau)=S_{f}(T-\tau)$ is as follows.

(1) Construct a division $0=\tau_{0}<\tau_{1}<\cdots<\tau_{m}=T$ of the interval [0,T]. To this end we can employ either an equidistant partition $\tau_{i}=(i / m) T$, or we can use $\tau_{i}=(i / m)^{2} T$ in order to adjust the discretization mesh to the desired behaviour (2.13) of $\varrho(\tau)$ close to expiry $\tau \approx 0$. We take $m \gg 1$ sufficiently large such that $(2 r / \sigma) \sqrt{2 \pi \tau_{1}} e^{r \tau_{1}}<1$.

(2) Compute the value of $\eta_{1} \approx \eta\left(\tau_{1}\right)$ from the analytical approximation formula (2.9), that is,

$$
\eta_{1}=-\sqrt{-\ln \left[\frac{2 r}{\sigma} \sqrt{2 \pi \tau_{1}} e^{r \tau_{1}}\right]}
$$

(3) For $i=2, \ldots, m$, compute the value $\eta_{i} \approx \eta\left(\tau_{i}\right)$ as follows.

(3-1) Construct the mapping $\mathcal{G}_{\eta_{i}}\left(\tau_{i}, \theta\right)=\left[\eta_{i}-\tilde{\eta}\left(\tau_{i} \sin ^{2} \theta\right) \sin \theta\right] / \cos \theta$, where $\tilde{\eta}\left(\tau_{i} \sin ^{2} \theta\right)$ is a linear interpolation function between the points $\left(\tau_{j}, \eta_{j}\right)$ and $\left(\tau_{j+1}, \eta_{j+1}\right)$ if $\tau_{j} \leq \tau_{i} \sin ^{2} \theta<\tau_{j+1}$ for some $1 \leq j<i$, and if $0<\tau_{i} \sin ^{2} \theta<\tau_{1}$ then $\tilde{\eta}\left(\tau_{i} \sin ^{2} \theta\right)$ is given by (2.9).

(3-2) Construct the mapping

$$
\mathcal{F}_{\eta_{i}}\left(\tau_{i}\right)=2 \int_{0}^{\pi / 2} e^{-r \tau_{i} \cos ^{2} \theta-\mathcal{G}_{\eta_{i}}^{2}\left(\tau_{i}, \theta\right)}\left(\frac{\sigma \sqrt{\tau_{i}}}{\sqrt{2}} \sin \theta+\mathcal{G}_{\eta_{i}}\left(\tau_{i}, \theta\right) \tan \theta\right) d \theta .
$$

For numerical quadrature of the above integral we can employ the composed Newton-Cotes method of fourth order with at least 1000 subintervals.

(3-3) Find the root $\eta_{i}$ of the equation

$$
\eta_{i}=-\sqrt{-\ln \left[\frac{r \sqrt{2 \pi \tau_{i}}}{\sigma} e^{r \tau_{i}}\left(1-\frac{\mathcal{F}_{\eta_{i}}\left(\tau_{i}\right)}{\sqrt{\pi}}\right)\right]}
$$


The above equation can be solved using either the bisection method, Newton's method, or any other numerical iterative method for finding roots of real-valued functions. In order to speed up convergence we can use the already constructed value $\eta_{i-1}$ as a starting point for iterations at time $\tau_{i}$.

(4) Go to step (3) and repeat the calculation of $\eta_{i}$ for the next value of $i$ such that $i \leq m$.

(5) From the discrete values $\eta_{i}, i=1,2, \ldots, m$, compute the approximation $\varrho_{i}$ of the early exercise boundary position $\varrho\left(\tau_{i}\right)$ as follows:

$$
\varrho_{i}=E e^{-\left(r-\sigma^{2} / 2\right) \tau_{i}+\sigma \sqrt{2 \tau_{i}} \eta_{i}}
$$

Set $\varrho_{0}=E$. The whole profile $\varrho(\tau)=S_{f}(T-\tau), \tau \in[0, T]$, is then computed as a linear interpolation function between the discrete values $\left(\tau_{i}, \varrho_{i}\right), i=$ $0, \ldots, m$.

3.2. Approximate solution using the PSOR method We present a brief overview of how the early exercise boundary can be found using a finite-difference numerical approximation method applied to the variational inequality (1.2). The method computes the option price $V(S, t)$ using the PSOR method introduced by Ockendon and Elliot [13]. Having computed a solution $V(S, t)$ to the variational inequality (1.2), we can calculate the early exercise boundary position. Indeed, given a time $t$, the critical stock price $S_{f}(t)$ is equal to the maximal stock price $S=S_{f}(t)$ for which the option price is equal to the payoff, that is,

$$
S_{f}(t)=\max \left\{S>0 \mid V(S, t)=(E-S)^{+}\right\} .
$$

Following Kwok [21], the idea of the PSOR method is to transform (1.2) by introducing new variables $x=\ln (S / E), \tau=T-t, u(x, \tau)=e^{\alpha x+\beta \tau} V\left(E e^{x}, T-\tau\right)$, where $\alpha, \beta$ are constants defined by

$$
\alpha=\frac{r}{\sigma^{2}}-\frac{1}{2}, \quad \beta=\frac{r}{2}+\frac{\sigma^{2}}{8}+\frac{r^{2}}{2 \sigma^{2}} .
$$

We denote by $u_{i}^{j} \approx u(i h, j k)$ the finite-difference approximation to a solution of the transformed variational inequality for $i=-n, \ldots,-1,0,1, \ldots, n, j=1, \ldots, m$. The spatial and time discretization steps $h, k>0$ are chosen such that $h=L / n$, $k=T / m$, respectively. Here $T$ represents the expiration time and $L$ is a sufficiently large bound for the interval $x \in(-L, L)$. For practical purposes we can take $L \approx 1$. In each time step $j=1,2, \ldots, m$, a linear complementarity problem for the finitedifference approximation vector $u^{j} \in \mathbb{R}^{2 n+1}$ is solved by using the iterative successive over-relaxation (SOR) method where iterates are projected to the transformed pay-off diagram. This is done by taking the maximum of the transformed pay-off and the SOR iteration. For details we refer the reader to [21, pp. 212-224]. 


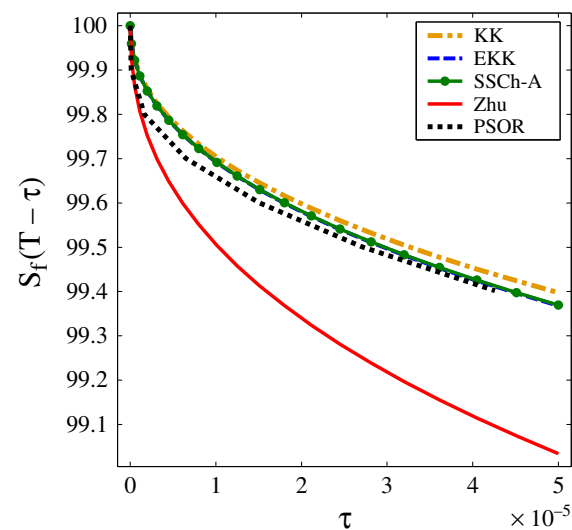

(a) $T=5 \times 10^{-5}(1 \mathrm{~min})$

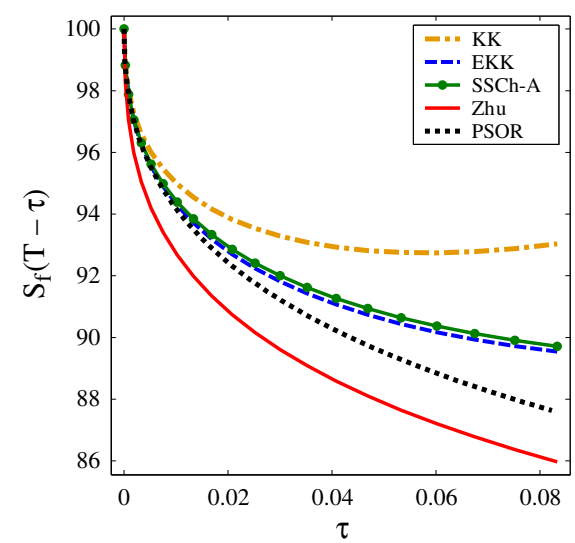

(c) $T=0.08(1$ month $)$

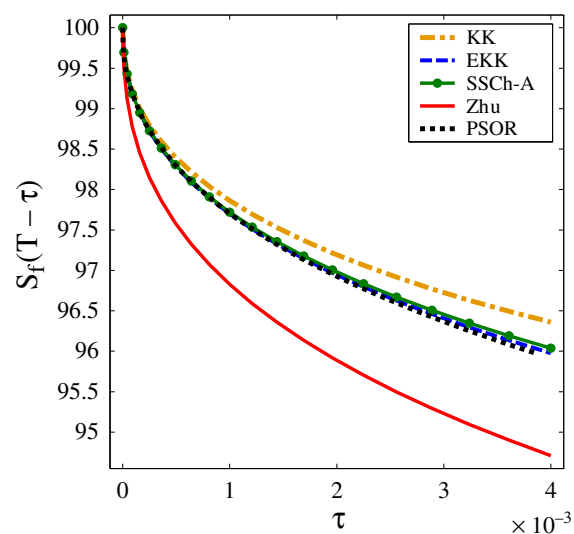

(b) $T=4 \times 10^{-3}$ (1 day)

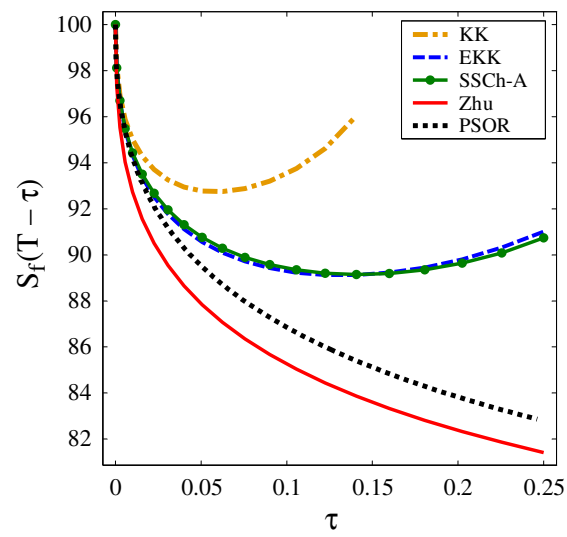

(d) $T=0.25$ (3 months)

FIGURE 3. Comparison of analytical approximation formulae for various maturities $T$ on a yearly basis.

\section{Numerical comparison of the early exercise boundary approximations}

4.1. Comparison of approximations close to expiry This section focuses on numerical comparison of the analytical approximations due to Stamicar et al. (2.5), Kuske and Keller (2.14), Evans et al. (2.15), Zhu (2.1) and our new local iterative algorithm from Section 3.1 for the early exercise boundary for times $0<\tau=T-t \ll 1$ close to expiry. For computational purposes we chose the volatility $\sigma=30 \%$, risk-free interest rate $r=10 \%$ p.a., and strike price $E=\$ 100$.

In Figure 3 we present a quantitative comparison of the analytical approximation formulae by Kuske and Keller (KK), Evans et al. (EKK), Stamicar et al. (SSCh-A) and Zhu. As a numerical benchmark solution we chose the PSOR method with $n=1000$ spatial grid points and $m=1000$ time steps (see Section 3.2). It should be obvious that the approximation formulae KK, EKK and SSCh-A exhibit similar behaviour with 
TABLE 1. Comparison of the early exercise boundary obtained by analytical approximation formulae and the iterative algorithm to the benchmark PSOR method.

\begin{tabular}{|c|c|c|c|c|c|c|c|c|c|}
\hline \multirow[b]{2}{*}{$\tau$} & \multicolumn{5}{|c|}{$\begin{array}{l}\text { Early exercise boundary } \\
\qquad \varrho(\tau)=S_{f}(T-\tau)\end{array}$} & \multicolumn{4}{|c|}{$\begin{array}{c}\text { Relative error } \\
\text { with respect to the PSOR method }\end{array}$} \\
\hline & EKK & Zhu & SSCh-A & SSCh & PSOR & EKK & Zhu & SSCh-A & SSCh \\
\hline 0.00001 & 99.69 & 99.51 & 99.69 & 99.69 & 99.70 & $0.01 \%$ & $0.19 \%$ & $0.01 \%$ & $0.01 \%$ \\
\hline 0.00005 & 99.37 & 99.03 & 99.37 & 99.36 & 99.40 & $0.03 \%$ & $0.37 \%$ & $0.03 \%$ & $0.04 \%$ \\
\hline 0.0001 & 99.14 & 98.72 & 99.15 & 99.11 & 99.20 & $0.06 \%$ & $0.49 \%$ & $0.06 \%$ & $0.09 \%$ \\
\hline 0.0005 & 98.28 & 97.57 & 98.29 & 98.27 & 98.31 & $0.03 \%$ & $0.76 \%$ & $0.02 \%$ & $0.04 \%$ \\
\hline 0.001 & 97.70 & 96.83 & 97.72 & 97.66 & 97.73 & $0.03 \%$ & $0.92 \%$ & $0.01 \%$ & $0.07 \%$ \\
\hline 0.01 & 95.62 & 94.27 & 95.69 & 95.50 & 95.60 & $0.02 \%$ & $1.39 \%$ & $0.09 \%$ & $0.10 \%$ \\
\hline 0.01 & 94.33 & 92.73 & 94.43 & 94.07 & 94.18 & $0.16 \%$ & $1.54 \%$ & $0.27 \%$ & $0.11 \%$ \\
\hline 0.04 & 91.12 & 88.66 & 91.31 & 90.21 & 90.30 & $0.90 \%$ & $1.82 \%$ & $1.12 \%$ & $0.11 \%$ \\
\hline 0.1 & 89.29 & 85.25 & 89.42 & 86.76 & 86.94 & $2.70 \%$ & $1.93 \%$ & $2.86 \%$ & $0.20 \%$ \\
\hline
\end{tabular}

Legend: EKK—Evans et al. [14], SSCh-A—Stamicar et al. [28], SSCh—our new local iterative algorithm from Section 3.1, Zhu-Zhu [30], PSOR—Projected SOR method [21].

respect to PSOR for time close to expiry (see Figure 3(a), (b)). On the other hand, on a larger time horizon KK, EKK and SSCh-A become nondecreasing and Zhu's formula better approximates the PSOR solution (see Figure 3(c), (d)). It is also worth noting that Zhu's formula undershoots the early exercise boundary for small values of $\tau$ when compared to KK, EKK, SSCh-A and PSOR. This phenomenon can be easily justified by calculating the limit

$$
\lim _{\tau \rightarrow 0^{+}} \frac{E-\varrho^{\mathrm{SSCh}-\mathrm{A}}(\tau)}{E-\varrho^{\mathrm{Zhu}}(\tau)} \sqrt{-\ln \tau}=1 .
$$

Recall that the notation SSCh-A stands for the analytical approximation formula (2.13) which is valid for time $t=T-\tau$ close to the expiration $T$. In what follows, we denote by SSCh the early exercise boundary postion obtained by our local iterative algorithm from Section 3.1.

In Table 1 we calculated the early exercise boundary position for EKK, Zhu, SSCh-A, PSOR and our new local iterative algorithm SSCh. We also calculated the relative error $\Delta^{\text {method }}(\tau)$, defined as

$$
\Delta^{\text {method }}(\tau)=\frac{\left|S_{f}^{\text {method }}(T-\tau)-S_{f}^{\mathrm{PSOR}}(T-\tau)\right|}{S_{f}^{\mathrm{PSOR}}(T-\tau)} \text { for } \tau \in[0, T],
$$

where $S_{f}^{\mathrm{PSOR}}$ is the early exercise boundary computed by the PSOR method. For $\tau \approx 1$ minute, the EKK, SSCh-A and PSOR methods have almost identical values (close to $\$ 99.40$ ) whereas Zhu's boundary position has been calculated as $\$ 99.03$. On the other hand, other approximations (EKK and SSCh-A) differ significantly from the early exercise boundary obtained by the PSOR method as we increase time 
TABLE 2. Comparison of the early exercise boundary on a long time horizon.

\begin{tabular}{crrrrrr}
\hline & \multicolumn{3}{c}{ Early exercise boundary } & & \multicolumn{2}{c}{$\begin{array}{c}\text { Rel. error with respect to } \\
\text { PSOR method }\end{array}$} \\
\cline { 2 - 3 } $\boldsymbol{T}$ & PSOR & Zhu & SSCh & & Zhu & SSCh \\
\hline 0.00 & 100.00 & 100.00 & 100.00 & & $0 \%$ & $0 \%$ \\
0.02 & 92.87 & 90.86 & 92.35 & & $2.16 \%$ & $0.56 \%$ \\
0.04 & 90.77 & 88.66 & 90.21 & & $2.33 \%$ & $0.62 \%$ \\
0.06 & 89.33 & 87.22 & 88.78 & & $2.37 \%$ & $0.62 \%$ \\
0.08 & 88.24 & 86.13 & 87.67 & & $2.39 \%$ & $0.64 \%$ \\
0.10 & 87.33 & 85.25 & 86.76 & & $2.38 \%$ & $0.65 \%$ \\
0.20 & 84.30 & 82.38 & 83.75 & & $2.28 \%$ & $0.65 \%$ \\
0.40 & 81.02 & 79.36 & 80.48 & & $2.05 \%$ & $0.66 \%$ \\
0.60 & 79.06 & 77.60 & 78.54 & & $1.85 \%$ & $0.66 \%$ \\
0.80 & 77.70 & 76.38 & 77.19 & & $1.70 \%$ & $0.66 \%$ \\
1.00 & 76.67 & 75.46 & 76.16 & & $1.58 \%$ & $0.66 \%$ \\
1.50 & 74.91 & 73.89 & 74.41 & & $1.37 \%$ & $0.67 \%$ \\
2.00 & 73.81 & 72.87 & 73.27 & & $1.27 \%$ & $0.73 \%$ \\
3.00 & 72.58 & 71.62 & 71.87 & & $1.32 \%$ & $0.97 \%$ \\
4.00 & 72.01 & 70.88 & 71.05 & & $1.58 \%$ & $1.34 \%$ \\
5.00 & 71.80 & 70.39 & 70.51 & & $1.96 \%$ & $1.79 \%$ \\
\hline
\end{tabular}

Legend: SSCh—our new local iterative algorithm from Section 3.1, Zhu—Zhu [30], PSOR—[21].

to expiration $\tau>0.02$. The relative error in the early exercise boundary position calculated by Zhu's formula with respect to the PSOR method is less than $2 \%$. The best approximation of the early exercise boundary has been achieved by our local iterative algorithm SSCh.

In summary, SSCh-A, KK and EKK are suitable for approximation of the early exercise boundary close to expiration, whereas, for a longer time horizon, it is recommended to use Zhu's approximation. The new local iterative approximation derived in Section 3.1 can be used for both small and large time.

4.2. The long-time horizon In the long-time horizon, that is, $\tau=T-t \approx 1$ year or more, we can no longer use the analytical approximations SSCh-A, KK, EKK designed for $0<\tau \ll 1$. These solutions lose monotonicity for $\tau \approx 0.1$ and even become undefined for large values of $\tau$ because of the sign change in the logarithm. This is why only Zhu's formula for the early exercise boundary (2.1) can be used in the long term. We compared Zhu's approximation with the two numerical methods described in Section 3. The first method is our new numerical method (labelled SSCh) based on the integral equation (2.6) which was described in Section 3.1. The second method is the classical PSOR method described in Section 3.2.

As a time horizon, we chose the large expiration time $T=5$ years. Other model parameters are the same as in the previous section: $E=\$ 100, \sigma=30 \%, r=10 \%$ p.a. The computational results are shown in Figure 4 (left) and Table 2. We observe that 

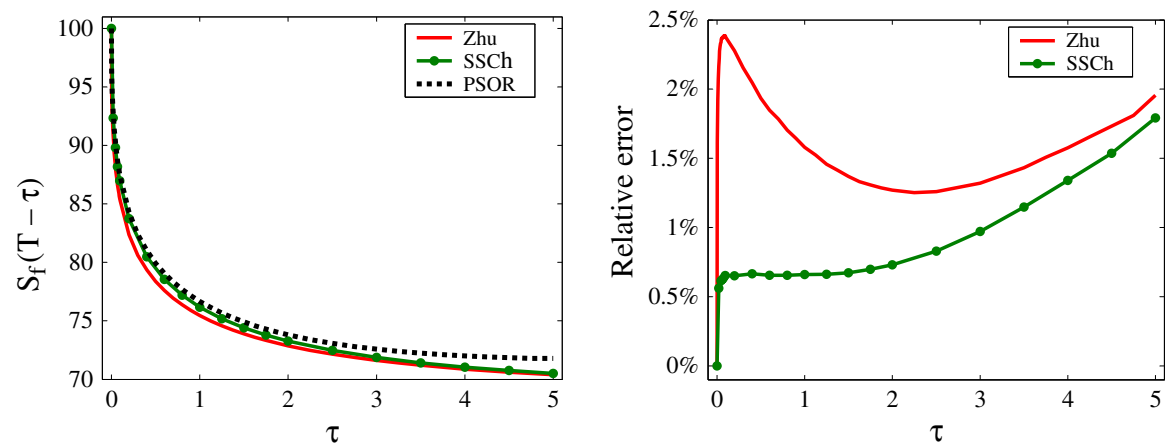

FIGURE 4. Comparison of the early exercise boundary position in the long time horizon. Early exercise boundary position (left) and the relative error with respect to the PSOR method (right).

the shape of all solutions is very similar. In Figure 4 (right) we plotted the relative error with respect to the PSOR method, which was used as a benchmark. Zhu's analytical approximation formula has relative error between $1 \%$ and $2.5 \%$ and attains a local minimum at around $\tau \approx 2$ years. This is due to the fact that Zhu's method is slightly undershooting the solution close to expiry, that is, for $\tau \approx 0$. The solution computed by our new SSCh scheme shows a nearly constant error term until $\tau \approx 2.5$ years, when the relative error starts to grow. For $\tau \approx 5$ years, the numerical solution SSCh is approaching Zhu's approximation. This is due to loss of precision in the PSOR method itself when the exact early exercise boundary could be closer to SSCh and Zhu's approximation than to the PSOR solution.

\section{Comparison of option prices}

In this section we address the difference between the American put option price and the approximate option price computed with an approximation of the early exercise boundary. More precisely, let $V^{\mathrm{am}}(S, t)$ be the solution to the free boundary problem (1.1) with the early exercise boundary profile $S_{f}$. Let us consider a given function $S_{f}^{\text {app }}$ representing an approximation of the early exercise boundary profile $S_{f}$. We denote by $V^{\text {app }}$ the unique solution to the parabolic equation

$$
\begin{gathered}
\frac{\partial V^{\mathrm{app}}}{\partial t}+r S \frac{\partial V^{\mathrm{app}}}{\partial S}+\frac{\sigma^{2}}{2} S^{2} \frac{\partial^{2} V^{\mathrm{app}}}{\partial S^{2}}-r V^{\mathrm{app}}=0, \quad t \in(0, T), S_{f}^{\mathrm{app}}(t)<S, \\
V^{\mathrm{app}}(+\infty, t)=0, \quad V^{\mathrm{app}}\left(S_{f}^{\mathrm{app}}(t), t\right)=E-S_{f}^{\mathrm{app}}(t), \\
V^{\mathrm{app}}(S, T)=(E-S)^{+} .
\end{gathered}
$$

Notice that we do not require the solution $V^{\text {app }}$ to satisfy the $C^{1}$ smooth pasting contact condition $\partial_{S} V^{\text {app }}\left(S_{f}^{\text {app }}(t), t\right)=-1$. In fact, $V^{\text {app }}$ is a solution to the put barrier option (see Kwok [21]) with a given down-and-out barrier $t \mapsto S^{\mathrm{app}}(t)$. For asset 


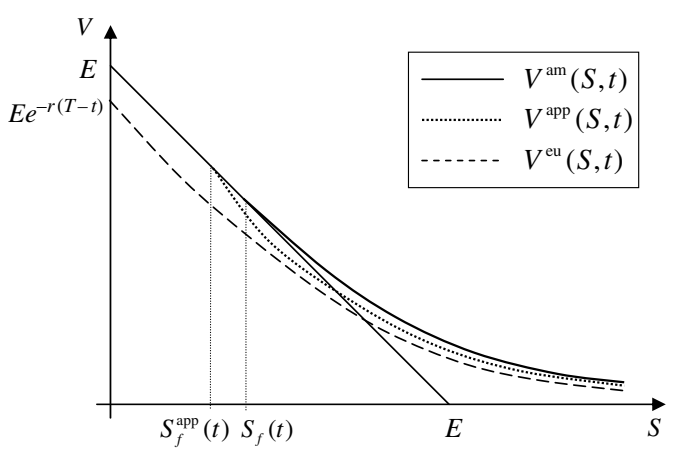

FiguRE 5. A profile $S \mapsto V^{\mathrm{am}}(S, t)$ of the American option price and comparison to the option price $V^{\text {app }}$ computed with respect to the approximate early exercise boundary $S_{f}^{\text {app }}(t)=\varrho^{\text {app }}(T-t)$. The corresponding European option is labelled by $V^{\mathrm{eu}}$.

prices $0<S<S_{f}^{\text {app }}(t)$ we set

$$
V^{\text {app }}(S, t)=E-S .
$$

A comparison of the profile $S \mapsto V^{\mathrm{am}}(S, t)$ of the American option price and the approximate option price $V^{\text {app }}$ is shown in Figure 5. We also plot the common lower bound for both put option prices represented by the European put option labelled by $V^{\mathrm{eu}}$.

Knowing the functions $t \mapsto S_{f}(t)$ and $t \mapsto S_{f}^{\mathrm{app}}(t)$, it is not difficult to calculate the difference $V^{\mathrm{am}}(S, t)-V^{\mathrm{app}}(S, t)$ between option prices. Indeed, using the standard transformation (see Kwok [21])

$$
V^{\mathrm{am}}(S, t)=E e^{-\alpha x-\beta \tau} u^{\mathrm{am}}(x, \tau), \quad V^{\mathrm{app}}(S, t)=E e^{-\alpha x-\beta \tau} u^{\mathrm{app}}(x, \tau),
$$

where $x=\ln (S / E), \tau=T-t$ (constants $\alpha, \beta$ are given in (3.1)) and taking into account the fact that $V^{\mathrm{am}}(S, t)=E-S$ for $0<S<S_{f}(t)$ and $V^{\mathrm{app}}(S, t)=E-S$ for $0<S<S_{f}^{\text {app }}(t)$, we conclude that $u^{\text {am }}, u^{\text {app }}$ are solutions to the following Cauchy problems:

$$
\begin{gathered}
\frac{\partial u^{\mathrm{am}}}{\partial \tau}-\frac{\sigma^{2}}{2} \frac{\partial^{2} u^{\mathrm{am}}}{\partial x^{2}}= \begin{cases}0 & \text { for } x>\ln (\varrho(\tau) / E), \\
r e^{\alpha x+\beta \tau} & \text { for } x \leq \ln (\varrho(\tau) / E),\end{cases} \\
\frac{\partial u^{\mathrm{app}}}{\partial \tau}-\frac{\sigma^{2}}{2} \frac{\partial^{2} u^{\mathrm{app}}}{\partial x^{2}}= \begin{cases}0 & \text { for } x>\ln \left(\varrho^{\mathrm{app}}(\tau) / E\right), \\
r e^{\alpha x+\beta \tau} & \text { for } x \leq \ln \left(\varrho^{\mathrm{app}}(\tau) / E\right),\end{cases}
\end{gathered}
$$

defined for $-\infty<x<\infty, 0<\tau<T$, where

$$
\varrho(\tau)=S_{f}(T-\tau), \varrho^{\mathrm{app}}(\tau)=S_{f}^{\mathrm{app}}(T-\tau) .
$$

Notice that the difference $v(x, \tau)=u^{\mathrm{am}}(x, \tau)-u^{\mathrm{app}}(x, \tau)$ satisfies $v(x, 0)=0$ for each $x \in \mathbb{R}$. Using Green's representation formula for a solution to a linear parabolic 
equation, we obtain, after some calculations, an explicit expression for the difference of option prices:

$$
\begin{aligned}
& V^{\mathrm{am}}(S, t)-V^{\mathrm{app}}(S, t) \\
& \quad=r E \int_{0}^{\tau}\left|\int_{\ln \left(\varrho^{\mathrm{app}}(\xi) / E\right)}^{\ln (\varrho(\xi) / E)} G(x-s, \tau-\xi) e^{-\alpha(x-s)-\beta(\tau-\xi)} d s\right| d \xi,
\end{aligned}
$$

where $G(x, \tau)=e^{-x^{2} /\left(2 \sigma^{2} \tau\right)} / \sqrt{2 \pi \sigma^{2} \tau}$ is the Green function. The above difference in option prices is always nonnegative because the American option price is greater than or equal to the price of a down-and-out barrier option with the prescribed barrier $S_{f}^{\mathrm{app}}(t)=\varrho^{\mathrm{app}}(T-t)($ see Kwok [21]).

If we evaluate this difference at the American option early exercise boundary position $S_{f}(t)$ then we obtain a slightly simplified expression:

$$
V^{\mathrm{am}}\left(S_{f}(t), t\right)-V^{\mathrm{app}}\left(S_{f}(t), t\right)=r E \int_{0}^{\tau} e^{-r(\tau-\xi)}|N(\tilde{\gamma}(\tau, \xi))-N(\gamma(\tau, \xi))| d \xi,
$$

where $\tau=T-t$ and

$$
\begin{gathered}
\tilde{\gamma}(\tau, \xi)=\frac{\ln \left(\varrho(\tau) / \varrho^{\mathrm{app}}(\xi)\right)+\left(r-\sigma^{2} / 2\right)(\tau-\xi)}{\sigma \sqrt{\tau-\xi}}, \\
\gamma(\tau, \xi)=\frac{\ln (\varrho(\tau) / \varrho(\xi))+\left(r-\sigma^{2} / 2\right)(\tau-\xi)}{\sigma \sqrt{\tau-\xi}} .
\end{gathered}
$$

Notice that the difference $V^{\mathrm{am}}(S, t)-V^{\mathrm{eu}}(S, t)$ of the American and European put options is rather small. Similarly, the difference $V^{\mathrm{am}}(S, t)-V^{\mathrm{app}}(S, t)$ is small. Therefore it is reasonable to calculate the mispricing error $V^{\mathrm{am}}(S, t)-V^{\mathrm{app}}(S, t)$ with respect to the benchmark mispricing difference $V^{\mathrm{am}}(S, t)-V^{\mathrm{eu}}(S, t)$ evaluated at $S=S_{f}(t)$. To this end, let us introduce the following relative mispricing error function:

$$
\operatorname{err}(T-t)=\frac{V^{\mathrm{am}}\left(S_{f}(t), t\right)-V^{\mathrm{app}}\left(S_{f}(t), t\right)}{V^{\mathrm{am}}\left(S_{f}(t), t\right)-V^{\mathrm{eu}}\left(S_{f}(t), t\right)} .
$$

The denominator of (5.4) can be easily calculated by recalling that

$$
V^{\mathrm{am}}\left(S_{f}(t), t\right)=E-S_{f}(t) \quad \text { and } \quad V^{\mathrm{eu}}(S, t)=E e^{-r(T-t)} N\left(-d_{2}\right)-S N\left(-d_{1}\right),
$$

where

$$
d_{1}=\frac{\ln (S / E)+\left(r+\sigma^{2} / 2\right)(T-t)}{\sigma \sqrt{T-t}}, \quad d_{2}=\frac{\ln (S / E)+\left(r-\sigma^{2} / 2\right)(T-t)}{\sigma \sqrt{T-t}}
$$

(see Kwok [21]).

In our practical experiment, we evaluated the relative mispricing error function $\operatorname{err}(\tau)$ for the approximation of the early exercise boundary obtained by Zhu, that 

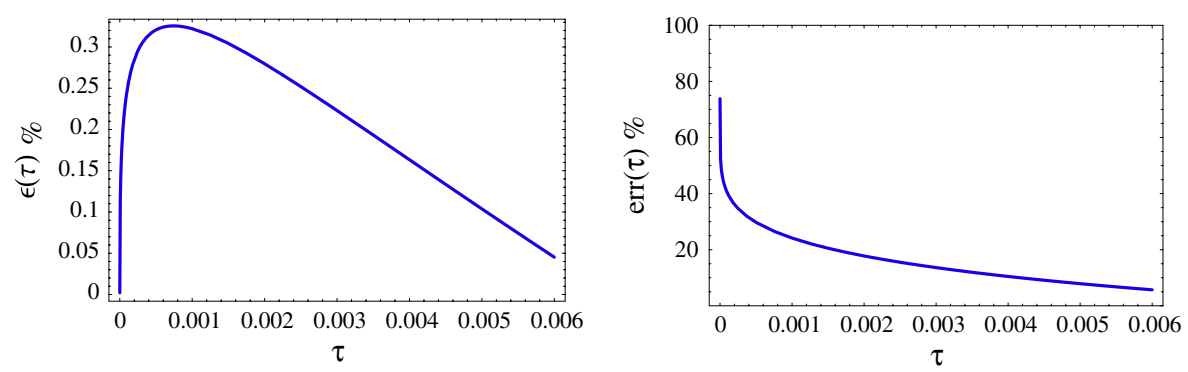

FIGURE 6. The relative error $\epsilon(\tau)$ in the early exercise boundary $\varrho^{\text {app }} \equiv \varrho^{\text {Zhu }}$ obtained from Zhu's formula (left). The relative mispricing error $\operatorname{err}(\tau)$ in the option price (right). The model parameters were chosen as $E=1, r=0.1, \sigma=0.3$ for the time $\tau=T-t \in(0,0.006)$ close to expiration.

is, we set $\varrho^{\text {app }} \equiv \varrho^{\text {Zhu }}$. In Figure 6 (left) we plot the relative error $\epsilon(\tau)$ in the early exercise boundary position

$$
\epsilon(T-t)=\frac{S_{f}(t)-S_{f}^{\mathrm{Zhu}}(t)}{S_{f}(t)}
$$

between the true early exercise position $S_{f}(t)=\varrho(T-t)$ and Zhu's approximation $S_{f}^{\mathrm{Zhu}}(t)=\varrho^{\mathrm{Zhu}}(T-t)$. We see that the maximal relative error in the early exercise boundary position is only $0.32 \%$ and it is attained 6 hours prior to expiration.

The relative error function $\operatorname{err}(\tau)$ for times $\tau=T-t$ close to expiry (less than 2 days) is depicted in Figure 6 (right). We see that the error rapidly increases when the time $t$ approaches expiration $T$. For 1 day to expiration $\left(\tau=4 \times 10^{-3}\right)$ the error is $15 \%$. It increases beyond $70 \%$ as $t \rightarrow T$. This is due to the fact that Zhu's approximation underestimates the free boundary position as $\tau=T-t \rightarrow 0^{+}$ (see (4.1)).

Based on our numerical experiments, we can draw the conclusion that the calculation of the free boundary position $S_{f}^{\mathrm{Zhu}}(t)$ using [30, Equation (2.24)] is still quite a reasonable approximation to $S_{f}(t)$ even when the time is very close to expiry. However, the errors in the calculation for the option price $V$ using [30, Equation (2.37)] can be quite large when $\tau=T-t \rightarrow 0^{+}$.

\section{Conclusions}

We have presented qualitative and quantitative comparisons of analytical approximations and numerical methods for computing the early exercise boundary position of the American put option paying zero dividends. We have also proposed a new local iterative numerical scheme for construction of the early exercise boundary which is based on a solution to a nonlinear integral equation. We have derived the asymptotic behaviour of approximation formulae for time close to expiry. We have proved that the formulae of Evans, Kuske and Keller [14, 20] and Stamicar 
et al. [28] have the same asymptotic behaviour close to expiry. We have also shown that the analytical approximation formula of Zhu [30] has a different asymptotic behaviour. On the other hand, for a long time horizon, Zhu's formula yields quantitatively the same results as those of our new local iterative numerical scheme and the numerical benchmark PSOR method. Our study has also revealed that the early exercise boundary analytical approximation from [14, 20, 28] and Zhu's approximation from [30] can be considered as complementary to each other in terms of providing good approximate solutions for small and large time to expiry, respectively.

\section{Acknowledgements}

We thank the anonymous referees for their valuable comments and suggestions. This research was supported by the VEGA 1/0381/09 grant and the bilateral SlovakBulgarian project APVV SK-BG-0034-08.

\section{References}

[1] G. Alobaidi, R. Mallier and A. S. Deakin, "Laplace transforms and installment options", Math. Models Methods Appl. Sci. 14 (2004) 1167-1189.

[2] J. Ankudinova and M. Ehrhardt, "On the numerical solution of nonlinear Black-Scholes equations", Comput. Math. Appl. 56 (2008) 799-812.

[3] G. Barone-Adesi and R. E. Whaley, "Efficient analytic approximation of American option values", J. Finance 81 (1987) 301-320.

[4] F. Black and M. Scholes, "The pricing of options and corporate liabilities", J. Political Economy 81 (1973) 637-654.

[5] D. S. Bunch and H. Johnson, "The American put option and its critical stock price", J. Finance 55 (2000) 2333-2356.

[6] P. Carr, R. Jarrow and R. Myneni, "Alternative characterizations of American put options", Math. Finance 2 (1992) 87-106.

[7] J. Chadam, "Free boundary problems in mathematical finance", in: Progress in industrial mathematics at ECMI 2006, Volume 12 of Math. Ind. (Springer, Berlin, 2008) 655-665.

[8] X. Chen and J. Chadam, "A mathematical analysis of the optimal exercise boundary for American put options", SIAM J. Math. Anal. 38 (2007) 1613-1641.

[9] X. Chen, J. Chadam, L. Jiang and W. Zheng, "Convexity of the exercise boundary of the American put option on a zero dividend asset", Math. Finance 18 (2008) 185-197.

[10] M. Ehrhardt and R. Mickens, "A fast, stable and accurate numerical method for the Black-Scholes equation of American options", Int. J. Theor. Appl. Finance 11 (2008) 471-501.

[11] E. Ekström, "Convexity of the optimal stopping boundary for the American put option", J. Math. Anal. Appl. 299 (2004) 147-156.

[12] E. Ekström and J. Tysk, "The American put is log-concave in the log-price", J. Math. Anal. Appl. 314 (2006) 710-723.

[13] C. M. Elliott and J. R. Ockendon, Weak and variational methods for free and moving boundary problems, Volume 59 of Res. Notes Math. (Pitman, London, 1982).

[14] J. D. Evans, R. Kuske and J. B. Keller, "American options on assets with dividends near expiry", Math. Finance 12 (2002) 219-237.

[15] R. Geske and H. E. Johnson, "The American put option valued analytically", J. Finance 39 (1984) 1511-1524.

[16] R. Geske and R. Roll, "On valuing American call options with the Black-Scholes European formula”, J. Finance 89 (1984) 443-455. 
[17] J. C. Hull, Options, futures, and other derivatives, 5th edn (Prentice Hall, Upper Saddle River, NJ, 2002).

[18] H. Johnson, "An analytic approximation of the American put price", J. Finan. Quant. Anal. 18 (1983) 141-148.

[19] I. Karatzas, "On the pricing American options", Appl. Math. Optim. 17 (1988) 37-60.

[20] R. A. Kuske and J. B. Keller, "Optimal exercise boundary for an American put option”, Appl. Math. Finance 5 (1998) 107-116.

[21] Y. K. Kwok, Mathematical models of financial derivatives (Springer, Singapore, 1998).

[22] Y. K. Kwok and L. Wu, "A front-fixing finite difference method for the valuation of American options", J. Financial Engineering 6 (1997) 83-97.

[23] L. W. MacMillan, "Analytic approximation for the American put option", Adv. Futures Options Res. 1 (1986) 119-134.

[24] R. Mallier and G. Alobaidi, "The American put option close to expiry", Acta Math. Univ. Comenian. (N.S.) 73 (2004) 161-174.

[25] R. Mynemi, "The pricing of the American option", Ann. Appl. Probab. 2 (1992) 1-23.

[26] D. Ševčovič, "Analysis of the free boundary for the pricing of an American call option", European J. Appl. Math. 12 (2001) 25-37.

[27] D. Ševčovič, "An iterative algorithm for evaluating approximations to the optimal exercise boundary for a nonlinear Black-Scholes equation”, Can. Appl. Math. Q. 15 (2007) 77-97.

[28] R. Stamicar, D. Ševčovič and J. Chadam, "The early exercise boundary for the American put near expiry: numerical approximation", Can. Appl. Math. Q. 7 (1999) 427-444.

[29] P. Wilmott, S. Howison and J. Dewynne, The mathematics of financial derivatives (Cambridge University Press, Cambridge, 1995).

[30] S. P. Zhu, "A new analytical approximation formula for the optimal exercise boundary of American put options", Int. J. Theor. Appl. Finance 9 (2006) 1141-1177.

[31] S. P. Zhu, "A simple approximation formula for calculating the optimal exercise boundary of American puts", J. Appl. Math. Comput. (2010) accepted for publication.

[32] S. P. Zhu and Z. W. He, "Calculating the early exercise boundary of American put options with an approximation formula", Int. J. Theor. Appl. Finance 10 (2007) 1203-1227. 